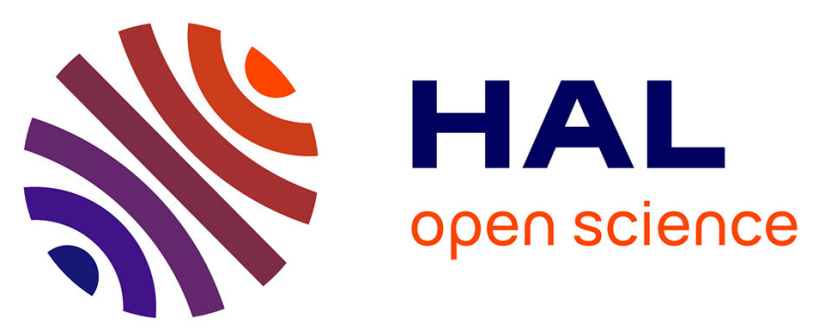

\title{
Virtual agent organizations for user behaviour pattern extraction in energy optimization processes: A new perspective
}

\author{
Alfonso González-Briones, Javier Prieto, Fernando de La Prieta, Yves \\ Demazeau, Juan Corchado
}

\section{To cite this version:}

Alfonso González-Briones, Javier Prieto, Fernando de La Prieta, Yves Demazeau, Juan Corchado. Virtual agent organizations for user behaviour pattern extraction in energy optimization processes: A new perspective. Neurocomputing, 2021, Vol. 452, pp.374-385. 10.1016/j.neucom.2020.05.117. hal-03046034

\section{HAL Id: hal-03046034 \\ https://hal.science/hal-03046034}

Submitted on 8 Dec 2020

HAL is a multi-disciplinary open access archive for the deposit and dissemination of scientific research documents, whether they are published or not. The documents may come from teaching and research institutions in France or abroad, or from public or private research centers.
L'archive ouverte pluridisciplinaire $\mathbf{H A L}$, est destinée au dépôt et à la diffusion de documents scientifiques de niveau recherche, publiés ou non, émanant des établissements d'enseignement et de recherche français ou étrangers, des laboratoires publics ou privés. 


\title{
Virtual agent organizations for user behaviour pattern extraction in energy optimization processes: A new perspective
}

\author{
Alfonso González-Briones ${ }^{\mathrm{a}, \mathrm{b}, \mathrm{c}, *}$, Javier Prieto $^{\mathrm{b}}$, Fernando De La Prieta $^{\mathrm{b}}$, Yves Demazeau ${ }^{\mathrm{d}}$, Juan M. Corchado ${ }^{\mathrm{b}, \mathrm{c}, \mathrm{e}, \mathrm{f}, *}$ \\ ${ }^{a}$ Research Group on Agent-Based, Social and Interdisciplinary Applications (GRASIA), Complutense University of Madrid, 28040 Madrid, Spain. \\ ${ }^{b}$ BISITE Research Group, University of Salamanca. Edificio I+D+i, Calle Espejo 2, 37007. Salamanca, Spain. \\ ${ }^{c}$ Air Institute, IoT Digital Innovation Hub, Carbajosa de la Sagrada, 37188. Salamanca, Spain. \\ ${ }^{d}$ University of Grenoble-Alps, CNRS-LIG, Grenoble, France \\ ${ }^{e}$ Department of Electronics, Information and Communication, Faculty of Engineering, Osaka Institute of Technology, $535-8585$ Osaka, Japan. \\ ${ }^{f}$ Pusat Komputeran dan Informatik, Universiti Malaysia Kelantan, Karung Berkunci 36, Pengkaan Chepa, 16100 Kota Bharu, Kelantan, Malaysia
}

\begin{abstract}
The optimization of energy use in family homes and public buildings is an ongoing topic of discussion. State-of-the-art research has almost always focused on reducing the consumption of heating systems, air-conditioning or lighting. Despite their importance, user-related variables, such as comfort, are normally not included in the optimization process. These aspects should be considered to be able to effectively minimize energy consumption. Thus, there is a need for a comprehensive energy optimization approach, one that will consider both climatological factors and user behaviour. Learning about user behaviour is key to effective optimization. In this work, the proposed architecture's capacity to organize Virtual Agent Organizations (VAO) allows it to adapt to highly variable user behavior and preferences. This agent methodology has the ability to manage Wireless Sensor Networks (WSNs), Artificial Neural Networks (ANN) and Case-Based Reasoning (CBR) to obtain user preferences and predict their behaviour in the home or building. The proposed approach has been tested in two different buildings, a traditional-construction house and a modular home, obtaining savings of $30.16 \%$ and $13.43 \%$, respectively. These results validate the proposed mixed approach of temperature adjustment algorithms together with the extraction of user behavior patterns for the establishment of a threshold based on preferences.
\end{abstract}

Keywords: Energy savings, Virtual Organization, CBR system, Sensor-based monitoring, Ambient Intelligent

\section{Introduction}

Climate change is currently one of the greatest concerns of governments around the world, given the dangers that climate change entails for our planet.The goal of the EU is to reduce greenhouse gas emissions by 80 $95 \%$ by 2050 compared to those in 1990 [1], [2].

EU leaders are committed to transforming Europe into a low carbon and high-efficiency economy. They establish actions that will help reduce the greenhouse effect and achieve high energy efficiency by reducing energy consumption in homes and public buildings [3]. Although it is a complex problem, everyone's individ-

\footnotetext{
${ }^{*}$ Corresponding author

Email addresses: alf fonsogb@ucm.es (Alfonso González-Briones), javierp@usal .es (Javier Prieto), fer@usal.es (Fernando De La Prieta), yves.demazeau@imag.fr (Yves Demazeau), corchado@usal.es (Juan M. Corchado)
}

ual effort, no matter how small, can contribute positively to minimizing this problem or transforming it altogether. Some of those measures simply involve raising awareness among the inhabitants about their use of energy at home and changing their habits to more energy-efficient. This can be achieved through recommendation systems which analyze user behavior data and suggest actions that will save energy.

The need to achieve greater energy efficiency has led to studies such as [4] which demonstrated that the annual energy bill can be reduced by $70 \%$ if appropriate energy-saving measures are taken. Heating and cooling systems are responsible for a large part of the overall energy consumed in homes and other buildings. In this research, the factors (environmental and human) that cause the systems' consumption to increase, have been identified. One of those factors is the importance of maintaining a steady level of temperature since dras- 
tic changes in temperature cause a significant increase in consumption. Moreover, to ensure user comfort, it is essential that the inhabitant' habits and preferences be taken into account when adjusting temperature in their homes. The correct regulation of the Heating, Ventilation and Air Conditioning (HVAC) system makes it possible to reduce energy bills and energy waste. When the temperature inside a home increases by one degree there is a 7\% increase in energy consumption, so appropriate temperature adjustments can help prevent drastic changes in temperature from one moment to another [5].

However, previous research works in this area have not taken into account the behavior of users which is at the center of energy consumption because many of the activities performed by users have a direct impact on the levels of energy being consumed [6]. As a result, it is necessary to develop a system that extracts user behavior patterns and establishes an ideal temperature according to this information. This enables the system to reduce the consumption of energy while meeting user preferences. Through the extraction of user behavior, the times at which users are present at home are determined, so that the temperature of heating can be programmed and adjusted to the schedules of the people who live in the house.

The objective of the presented proposal is to optimize the use of energy and satisfy the user by correctly adjusting a building's temperature, [7]. This will be achieved through the analyses of the main parameters that influence the consumption of energy in homes and buildings, related not only to the monitoring of environmental factors but also to the extraction of user behaviour characteristics. The architecture is based on a system of virtual organizations which coordinate their agents to make energy-efficient decisions. The decisionmaking process involves analyzing the information that is provided by the deployed sensor network, including temperature and presence data. This WSN also facilitates communication between the different devices that interact with the system's agents.

This proposal's main contributions include: extraction of user behavior patterns, which are used by the intelligent temperature adjustment algorithm; an energy consumption prediction algorithm that predicts if the temperature values established by the intelligent temperature adjustment algorithm are going to reduce energy consumption; the architecture's agents execute all activities, this means that they are automated and there is no need for the user to intervene at any stage of the process.

The motivation behind this work is to develop an architecture based on virtual organizations of agents, that considers user habits and preferences as well as the environmental variables that affect the consumption of energy. Thus, this architecture, will cover the shortcomings of the state-of-the-art described above [8].

This article is organized as follows: section 2 describes the state-of-the-art literature on energy optimization, Section 3 outlines the proposal, Section 4 presents the results and Section 5 the conclusions.

\section{Artificial intelligence for optimized energy use.}

This section outlines the technologies and the methodologies that have been used in previous architectures based on virtual organizations of agents for intelligent adjustment of temperature in buildings and homes. The following subsections review the current state of the techniques used by systems designed to save energy.

\subsection{Factors involved in the consumption of energy}

User activity is the main energy consumption factor in any home or public building. However, factors other than user preferences and habits can directly influence the behavior of users, including climatic factors (outdoor temperature, outdoor lighting, humidity, etc.), social factors (price of energy, environmental/social awareness, etc.) or emotional factors (joy, sadness, etc.). Until now, energy optimization proposals for homes and buildings have focused exclusively on climatic factors.

For this reason, the current research trend in the area of energy optimization is not only to focus on climatic factors but also on the patterns in the inhabitants' behavior [9]. This dual approach makes it possible to design systems driven by artificial intelligence algorithms that adjust the temperature according to a user behaviour model. In the energy balance of a building, the influence exerted by the user is clearly decisive, as a result some research works analyzed the users' lifestyle and their temperature preferences in order to program these systems and accommodate them to the needs of their users [10].

A research by Hoes et al. [11] studied the human activity parameters that influenced consumption, especially the opening of windows such as the season, outdoor and indoor temperature, time of day, presence. However, the results of this study cannot be applied to any building in general since these variables are for a specific type of building, location, climate or culture. Harris and Cahill [12] proposed the CAPM framework for effective energy management, it uses Bayesian networks to predict user behaviour patterns from multimodal sensor data. 
Therefore, an automated system must be developed in order to independently obtain the variables that influence energy consumption (acquisition of environmental data and user data), taking into account the presence of people in the building.

\subsection{Context and User behaviour pattern extraction}

There are many factors that are related to the consumption of energy in buildings and homes. The three major factors in energy consumption are climate, environmental characteristics and user behavior [13]. However, user behaviour is the most influential factor because ultimately, it is the inhabitants' activity that causes energy consumption to increase or decrease. Users may decide, for example, to raise or lower the temperature of the heating or the air conditioning systems, to turn the electrical devices on/off (lighting, oven, etc.). In this respect, it is fundamental to obtain not only information about the climate and the environment, but also to extract user behavior patterns. Thanks to the extraction of user behavior and knowledge of the times at which each user is in the home or building, it is possible to intelligently adjust the temperature.

To measure the values of those factors and learn about the behaviour of users, it is necessary to deploy a sensor network in the building or home. These networks acquire and send values to the platform, where they are used by decision making and temperature adjustment algorithms. To extend the monitoring of parameters to other rooms or homes at any time, the sensor system must be scalabe so that it will be possible to add new sensors easily. To learn about the behavior of users, some authors have proposed the use of motion sensors, acoustic sensors and presence sensors [14], so as to obtain information about the presence or absence of people in the home. Thus, a network of distributed sensors is an essential component for data collection and analysis in a home or a building.

WSNs provide parameter values and directly incorporate them into other devices within the system that need this information to perform their tasks (weather forecasting, learning user behavior, predicting energy consumption or temperature adjustment). These WSNs have been used in proposals that require the deployment of a large number of sensors, often the number of sensors is not known, examples of such works include [15], [16] and [17].

Apart of the information that the system acquires through the sensors, the system must be able to predict future conditions that will help it adjust its values before the actual information is available. This includes weekly temperature forecasts that can be obtained through the APIs of platforms like OpenWeatherMap or the State Agency of Meteorology (AEMET).

Although energy optimization architectures can obtain valid data through APIs, some authors have discovered that greater precision is achieved if the context information from the case study is used instead to predict future conditions [18], [19].

Neural networks are a computational model that has been widely used in the literature for the analysis and recognition of patterns based on similar data. Multiple researches have employed this model to classify the fatigue of computer-person interaction [20], firearm classification [21], cattle detection [22].

The Multilayer Perceptron (MLP) is a model of neural networks that bases its classification capacity on linearly dependent sets, this means that MLP is a suitable approach for forecasting weather when trained with previous weather data of similar characteristics, i.e. to forecast the weather in a particular place for a given month, it is necessary to train the MLP with data from the same place and period. It is good to train MLP with data from the last thirty years, to prevent any deviation in temperature, humidity or precipitation, due to the changes in weather.

Numerous approaches have also been adopted in the extraction of the inhabitants' behaviour patterns, such as finding out when users return to their home, or how many hours they spend outside or inside the household. There are many researches in the literature focused on the modeling and prediction of occupation in buildings and very diverse techniques have been used for this purpose. The review by Yang et al. shows the main models for performing occupancy modeling such as Markov model [23], Presence model-stochastic processMarkov chain [24], Markov Chain [25], Probabilisticbased model [26] or Non-probabilistic model [27].

However, these works have a high error rate in the modelling of user behaviour, it is necessary to know when there will be human presence in the home in advance. Case-based Reasoning (CBR) systems perfectly suit the needs of our problem; a CBR system stores all the past problems it has solved successfully and uses their solutions to find a solution to a new, similar problem. That is to say, once user behavior parameters are known, it is possible to predict their future behavior in the home; when they are present/absent from their home and at what times they enter/leave. To this end, it is necessary to model cases for each user with the following behavior variables: i) time at which they leave the dwelling, ii) time at which they enter the dwelling, iii) time spent outside the dwelling, iv) time spent inside the 
dwelling, v) whether it is a working day or not, vi) time of day, and vii) the period spent outside the dwelling on that day.

\subsection{Energy Consumption Prediction}

There are a number of techniques for predicting and estimating future values. In the field of energy optimization they have been used to calculate the energy demand of buildings or homes [28]. In this paper, prediction will be used to validate whether the decisions of intelligent temperature adjustment algorithms produce the desired results (reduced energy consumption).

In the literature, there have been many techniques that use a time series and then intend to estimate how the value of a variable is going to evolve by studying previous values and the pattern of evolution of the variable.New techniques and more evolved models have been developed on the basis of time series and information obtained through data mining techniques [29]. Some of these techniques make use of neural networks such as MLP [30], [31] and RBF (Radial Basis Function) [32], [33], regression techniques such as ARIMA ( $\mathrm{Au}-$ toRegressive Integrated Moving Average) [34], [35], SVR (Support Vector Regression) [36], [37], fuzzy networks and [38] or expert systems and hybrid methods [38], [39], [40] or Linear Regression (LR) [41] among others. The prediction of consumption in buildings and dwellings makes it possible to estimate the future evolution of a variable that is linked to energy consumption, this means that possible peaks can be foreseen and energy consumption can be predicted for a particular day in the future. In the case of electricity consumption, prediction is an essential tool to be applied to various case studies.

As mentioned previously, energy consumption in buildings is influenced by certain environmental variables, which must be considered if precise consumption prediction is to be achieved [42], [43].

Therefore, the analysis of the environmental variables will allow to incorporate important information into the models in order to improve the prediction results. There are multiple algorithms such as Linear Regressions (LR), Support Vector Regression (SVR), Gaussian Process Regression (GP), Random Forests (RF), or KNearest Neighbours (KNN) to perform such processes with a high degree of precision. In the work of Yan et al. several highly accurate methods are compared [44]. Therefore, a review of these methods will be carried out so that their implementation is integrated in the system to predict energy consumption in homes. Predicting energy consumption makes it possible to validate whether the decisions taken by the rest of the architecture will have the ideal results in terms of reducing energy consumption.

\subsection{Distributed Architectures}

A distributed architecture is essential if a priori the number of deployed sensors and IoT devices is not known, it must also have the capacity to incorporate acquisition systems or analysis techniques autonomously and dynamically, without endangering the stability of the system at any time. Like in any distributed architecture, the relationship between the systems' agents and their communication with the rest of the elements in the system is intrinsically complex to model and design.

However, agent systems also have many advantages that make them an ideal approach to problems like the one presented in this research. The agents that make up this type of architecture have a shared goal and a protocol that allows them to collaborate with each other. They group activities according to their core competences. They work together in teams (called layers in multi-agent systems; organizations in virtual organizations of agents) in order to bring their core competences to the value chain. They process and distribute the information throughout the whole system in real time, making decisions and coordinating the rest of the agents. These architectures make it possible to aggregate new agents to the system whenever new competences are required, all this can be done without stopping the operation of the system.

Furthermore, the autonomy with which the agent systems are equipped enables them to interact with each other without human intervention. Their ability to perceive and react to changes in the environment makes this methodology an ideal approach for obtaining environmental data and responding to these changes with appropriate actions. Features such as extensibility and flexibility make it possible to add new features or include other algorithms and sensors.

One of the disadvantages is that there is no standard methodology to model MASs, so it is necessary to evaluate the different methodologies in order to choose the one that best suits the present work.

MAS design and modelling is undergoing diverse changes. This has resulted in multiagent systems that are much more evolved than the initial ones. This has led to the emergence of systems based on virtual organizations of agents, which introduce concepts derived from the social organization, such as roles, groups and rules. This enables VOs to operate in highly dynamic environments more efficiently. Unlike multi-agent systems, in virtual organizations agents are free to move between organizations and can take on a different roles 
if required, moreover, organizations can be cloned if a larger number of the same type of agents is required.

The roles are assigned to agents according to their capacities because these systems must be able to adapt to highly dynamic, real environments. The change of an agent from one organization to another entails a reorganization and restructuring of the agents within the virtual organizations that compose the system. In addition, since the paradigm of virtual organizations is based on human societies, it is necessary for the architecture to have a series of rules, for when and how reorganizations are to be performed and under what circumstances. These rules will ensure that the process of reorganization is efficient [45].

Agent-based systems have often been applied in the field of building automation due to their ability to monitor large surfaces through the deployment of new agents. It can be concluded that this approach facilitates the growth of simple systems into more complex ones, either through the deployment of new agents, such as sensor control agents, communication agents, data analysis agents or decision-making agents.

These advantages have led McArthur et al. [7], [46] to apply multi-agent systems to the problem of energy wastage produced as a result of drastic changes in temperature. Virtual agent organizations have also been used for this purpose, although the developed system was more simple [47]. Other proposals based on agent systems focused on adjusting temperature in HVAC systems for optimized energy use [48]. An outstanding proposal that can be found in the literature is that of Wang et al. who used an agent-based system that implements a control system with an intelligent temperature optimizer. The developed system effectively managed the consumption of energy while ensuring a degree of comfort for the inhabitants of a house or a building. The goal of their work was to achieve energy efficiency through the deployment of sensors and an agent architecture, in this way creating intelligent buildings [49]. Another remarkable work is that carried out by Cai et al. whose agent system managed the central air conditioning systems of a building [50]. In this work, the optimization problem was reformulated as several subproblems, each of which was solved by an individual agent.

However, the success rate of th(averages are obtained of all the values collected by all the sensors. The systems described above would have been higher if the developed architectures had been implemented using virtual agent organizations. This is because, virtual agent organizations would have provided a range of solutions for each of the sub-problems of the optimization prob- lem, allowing the choice of the solution that offers the best results and the implementation of different artificial intelligence techniques. One important proposal that leverages Virtual Organizations in an energy optimization problem is the one proposed by Al-Daraiseh et al. whose system focuses on optimizing energy consumption in educational institutions by predicting the periods in which HVAC systems were turned on and off [6]. In that paper, the authors considered the influence of certain factors, such as external climatic conditions and the presence of people. However, the proposed system lacked automation and the values had to be entered manually, making it impossible to achieve visible energy savings.

The shortcomings of state-of-the-art research, identified in the area of energy optimisation, make evident the need for an architecture based on virtual agent organisations. This architecture is going to cover the current research gap by providing several solutions for intelligent temperature adjustment in the home, for greater comfort and energy optimization. These deficiencies can be solved through the design of an architecture based on virtual organizations that model a MAS following the structural model of human organizations. The use of this kind of agent structure allows us to limit the unpredictability of the system within a grouping of agents subject to a series of defined roles. VOs try to delimit some problems of autonomy of the agents by grouping them within layers in the MAS. These complex systems depend largely on external conditions and the needs of end users as is the case of the problem that in this article is raised in which it is intended through virtual organizations of agents to collect the values of parameters that influence energy consumption and thus adjust the temperature intelligently to reduce energy consumption.

An example of a system whose aim is similar to that of the research presented in this article, is that of Claus et al. [51] where virtual organizations are used to integrate distributed energy resources agents in virtual power plants (VPP). The approach models distributed energy resources and virtual power plants as agents with multi-objective reasoning and of multiple questions. This allows the VPP's shaped one that resources of energy constitute distributed complex and heterogeneous with multiple local aims and points of decision. This approach is similar to ours however, our vision is to create a system that users are going to be able to manage effectively. To this end, our approach relies on organizations of agents which obtain the users' preference values, indoor and outdoor temperature of a building and detecting the presence of inhabitants. 


\section{Overview of the proposed architecture}

This section details the technical aspects of the proposed agent-based architecture, related to the collection of data through sensors, weather forecasts, extraction of user characteristics such as daily schedule, intelligent temperature adjustment algorithm and the temperature adjustment decision validation system for effective energy saving.

\subsection{Sensor deployment and data acquisition}

The sensors implemented by the system detect the presence of people in the home/building and collect outdoor and indoor temperature values.

- Amperimetric clamp: The Energy Meter Gen5 (Aeotec) https://aeotec.com/ z-wave-home-energy-measure measures the voltage in the $\mathrm{AC}$ and $\mathrm{DC}$ ranges and obtains consumption measurements in real time with an accuracy of $99 \%$. Measurements are sent via wireless connectivity that is optimised to transmit over greater distances and even through walls. Without interference, Home Energy Meter can transmit a home's electricity use over a distance of 492 feet / 150 metres with the data securely broadcast using AES-128 encryption.

- Smart thermostat: The Secure Controls SRT 323 Thermostat https://www.securemeters. com/index.php/products/residential/ home-heating-hot-water-controls/ room-thermostats/srt323-z-wave/ has been used. This thermostat can directly replace traditional thermostats and there is no need to change the wiring. As a result of this device, the heating adjustment value is sent remotely through the Z-Wave gateway.

- Multisensor: The Aeotec MultiSensor 6 (Motion, Temperature, Lighting, Humidity, Vibration and UV) https://aeotec.com/z-wave-sensor has been used to obtain the value of the variables with which it predicts meteorological conditions (Temperature, Lighting, Humidity and UV) and to extract user behavior patterns (Motion and Vibration).

- Opening sensors: The Aeotec Door/Window Sensor Gen5 https://aeotec.com/ z-wave-door-window-sensor has been used to detect open doors or windows. It is used jointly with the multisensor to detect presence in houses or buildings. This value is used to extract users' behavior pattern.

- Smart Plug: The Fibaro Wall Plug https:// www.fibaro.com/en/products/wall-plug/ has been used, it is a remotely controlled plug-in switch with the ability to measure power and energy consumption. It uses a LED frame to visualize the current load and operating mode with color changing lighting. This smart plug makes it possible to control electrical devices in a convenient and maintenance-free way.

- Gateway Z-wave: The Gateway is the intermediary between the previously described devices and the system that implements the proposed architecture. The Gateway acts as a communication link between devices and the platform.

For the system to correctly adjust temperature and take the appropriate energy optimization decisions, it is necessary for it to have data on the presence of people in the home, indoor and outdoor temperature and weather forecasts. Prior knowledge of those factors is essential. If the system knows the times at which the inhabitants will be present at home, it will be able to regulate the temperature accordingly; lower the temperature or turn off the radiators when inhabitants away. However, the system will not switch off all the heating since the cooler the home gets the more energy is required to heat the house before the inhabitants return, causing greater consumption than if an optimal temperature was maintained.

\subsection{ANN for weather forecasting}

The system has an agent in the AI Analysis VO layer which forecasts the weather. Temperature is adjusted on the basis of the data this agent provides. It forecasts weather data for the next thirty days. It models an algorithm that uses an ANN, specifically, a Multi-Layer Perceptron (MLP) as shown in Figure 1 with a Sigmoid activation function and the Resistant Propagation function used as the training method.

The agent in charge of forecasting weather for the next month (thirty days), trains the MLP with the meteorological data for that month from the last two years and with data from the last thirty days. These data are provided in Table 4. This makes it possible to obtain a predictable weather forecast for the next thirty days. Training data must be in CSV format with the following header: temp $p_{\max } 1$, temp $p_{\min } 1$, wind $_{\max } 1$, wind $\min 1$, $p_{\text {avg }} 1, R H_{\text {avg }} 1, S R_{\text {avg }} 1$, temp $_{\max } 2$, temp $_{\text {min }} 2$, wind $_{\text {max }} 2$, 


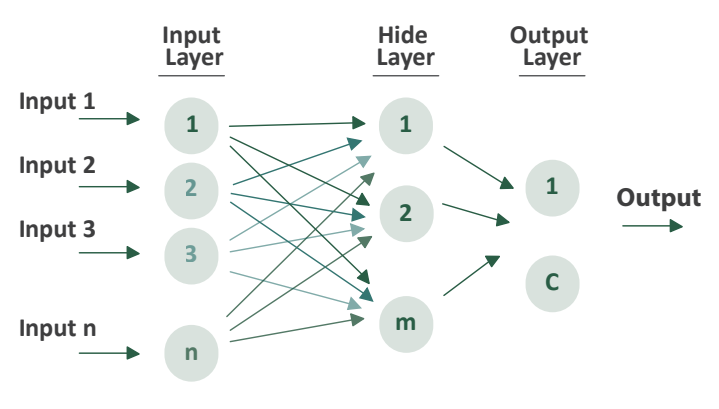

Figure 1: MLP structure for weather forecasting

wind $_{\min } 2, \quad p_{\text {avg }} 2, \quad R H_{\text {avg }} 2, \quad S R_{\text {avg }} 2, \ldots, \quad$ temp $p_{\text {max }} 30$, temp min $_{\text {min }} 30$, wind $_{\max } 30$, wind $_{\min } 30, p_{\text {avg }} 30, R H_{\text {avg }} 30$, $S R_{\text {avg }} 30, \quad \ldots, \quad$ temp $_{\text {max }} 60, \quad$ temp $_{\text {min }} 60$, wind $_{\text {max }} 60$, wind $_{\text {min }} 60, p_{\text {avg }} 60, R H_{\text {avg }} 1, S R_{\text {avg }} 60, \ldots$, temp $_{\text {max }} 90$, temp min $_{\text {min }} 90$, wind $_{\max } 90$, wind $_{\min } 90, p_{\text {avg }} 90, R H_{\text {avg }} 90$,

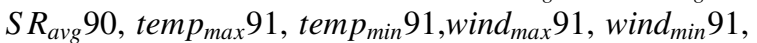
$p_{\text {avg }} 91, R H_{\text {avg }} 91, S R_{\text {avg }} 91$. Where the first thirty values correspond to the values obtained over thirty days, two years ago(1-30), the next thirty values correspond to the previous year (30-60) and the last thirty values correspond to the last thirty days (60-90) and temp $p_{\max } 91$, temp $_{\min } 91$, wind $_{\max } 91$, wind $_{\min } 91$, p avg $_{\text {av }} 91, R H_{\text {avg }} 91$, $S R_{\text {avg }} 91$ are the values predicted by the MLP. To prevent the architecture from suffering cold starts (lack of data), Weather_history_agent provides weather data by checking the weather history data on Weather Underground (https://www . wunderground.com/history/). The system trains the algorithm with data for the period and city selected in the system. These two parameters can be configured by the inhabitants themselves.

Table 1: Variables used in the MLP training. The displayed sensors collect the value of each variable every hour.

\begin{tabular}{lll}
\hline Variable & \multicolumn{1}{c}{ Description } & \multicolumn{1}{c}{ Units } \\
\hline temp $_{\max }$ & Maximum temperature & ${ }^{\circ} \mathrm{C}$ \\
temp $_{\min }$ & Minimum temperature & ${ }^{\circ} \mathrm{C}$ \\
wind $_{\max }$ & Maximum wind speed & $\mathrm{km} / \mathrm{day}$ \\
wind $_{\min }$ & Minimum wind speed & $\mathrm{km} / \mathrm{day}$ \\
$p_{\text {avg }}$ & Average precipitation & $\mathrm{mm}$ \\
$R H_{\text {avg }}$ & Average relative humidity & $\%$ \\
$S R_{\text {avg }}$ & Average solar radiation & $\mathrm{MJ} / \mathrm{m}^{2} / \mathrm{day}$ \\
\hline
\end{tabular}

\subsection{CBR system for learning user behaviour}

A Case Based Reasoning System (CBR) learns user behaviour with the data obtained by the sensor network. A CBR system learns schedules automatically from the cases collected in the baseline period and thus obtains or predicts the future behaviour of users on the basis of their past behaviour (when users leave the home and when they come back). In the literature, agent architectures have been identified of systems for similar purposes [5].

The architecture has an agent that implements a CBR system within the virtual organization. This agent performs the extraction of user characteristics through the deployed sensor network, that is, it obtains information about the times at which users are present in the house, at what times they leave and enter and for how long they stay. This feature extraction process allows to create the cases with which the CBR will perform different models of user behavior (information and schedules).

Once the different user schedule models have been created (that is to say, the cases of the CBR have been constructed) for a set of users living in a house, the database that stores the cases with which the CBR works, is constructed to learn and predict the predominant schedules and to send this information to the intelligent temperature adjustment algorithms (The schema of the developed CBR is shown in Figure 2).

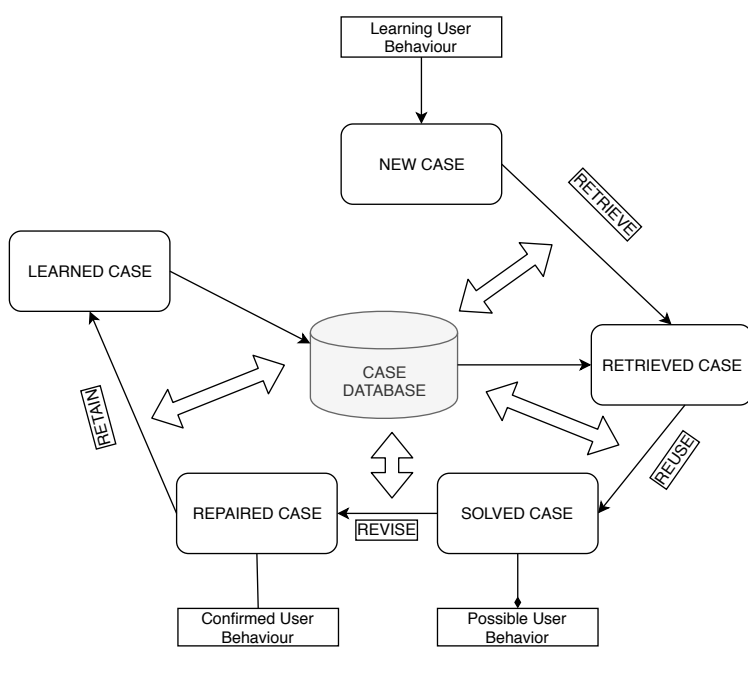

Figure 2: The CBR Cycle according to Aamodt and Plaza [52].

To create models of schedules for a group of users living together in a dwelling,CBR systems carry out a process based on four different sub-processes (Retrieve, Reuse, Review, Retain). Retrieve: the system retrieves cases that are similar to the current case (cases that share values of similar variables) in order to resolve it. Each of the recovered cases contains the values of its variables, the solution to the problem and possible annota- 
tions on the solution. A Euclidean distance-based algorithm has been used to extract cases, as shown in 1

$$
d=\frac{\sum_{i=1}^{n} w_{i} \operatorname{sim}\left(f_{i}^{I}, f_{i}^{R}\right)}{\sum_{i=1}^{n} w_{i}}
$$

Where:

- $i$ variables $\left(\right.$ time $_{\text {now }}$, time $_{\text {leave, }}$, time $_{\text {leavePeriod }}$, time $_{\text {back }}$, time $_{\text {backPeriod }}$, time $_{\text {period }}$, time $\left._{\text {periodDay }}\right)$;

- wi the weight of the $i$ th variable;

- $f_{R}^{I} i$ th variable value for the input case;

- $f_{i}^{R} i$ th variable value for the retrieved case;

- $\operatorname{sim}\left(f_{R}^{I}, f_{i}^{R}\right)$ the similarity between $f_{R}^{I}$ and $f_{i}^{R}$.

This is done in the first stage of the CBR process. Each of these stages is detailed below. Reuse: The solution obtained in a similar past case is chosen as a basis for the solution of current problem. At this stage it is possible that the solution must be adapted to the new situation. Review: Once the solution to the current problem has been assigned, the new solution is tested in a simulation process in order to find out if it requires revision. Retain: Once the solution has been successfully applied to solve the current problem, a new case is stored in the database, with the values of the variables, adopted solution and notes about the solution.

\subsection{Intelligent temperature adjustment algorithm}

The intelligent temperature adjustment algorithm is fed with the data acquired by the system, based on the current environmental information, user characteristics, the weather forecast, that is, the factors that must be considered when adjusting temperature in the home. The algorithm provides the temperature value that must be set on the thermostat to achieve a reduction in energy consumption. The algorithm makes decisions about the temperature adjustment and the switching off and on of the HVAC system or the devices connected to the smart plugs. The use of smart thermostats allows to modify the value of the temperature set in the home, minimizing sudden temperature changes (an increase or decrease by several degrees within a short period of time, these changes are the ones that the architecture avoids in order to reduce the consumption of unnecessary energy). The developed algorithm takes advantage of the knowledge of all the factors thanks to the communication between virtual organizations, as well as of the data provided by the MLP in terms of meteorological prediction or by the CBR in order to know when the residents leave home every day, time $_{\text {leave }}$, and the time that elapses until they return time $_{\text {back }}$, also the time that elapses inside the home time $_{\text {backPeriod. }}$ Temperature parameters are obtained by sensors, temperature variables include indoor $t m p_{\text {indoor }}$ and outdoor temperature $t m p_{\text {outdoor }}$, desired temperature $t m p_{\text {desired }}$ and forecast temperature $t m p_{\text {forecast }}$. The system must also consider whether air conditioning units and radiators are switched on or off, $t m p_{\text {status. }}$. The temperature adjustment period of the HVAC system will increase or decrease the temperature according to the inhabitants' preferences.

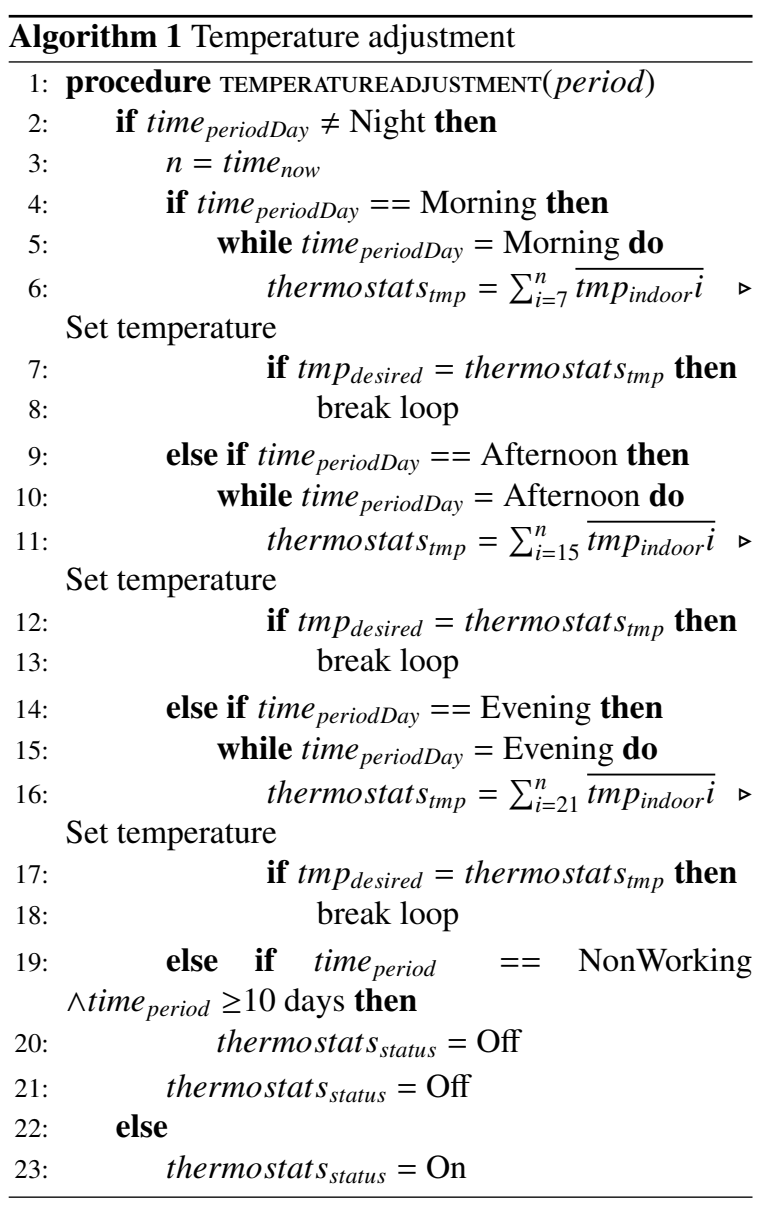

As observed in Algorithm 1, first, the algorithm considers the time of day and formats this value to a float number in order to set the variable $n$ to this value, which will be used to exit the temperature adjustment loops. The output of the loop also occurs if the temperature is increased by one degree every hour, reaching the temperature desired by users. The algorithm takes into account serveral aspects, such as, whether it is a non- 
Table 2: Variables used in CBR cases. The value of each variable is collected every day from the sensors, except time period, which is established by the system.

\begin{tabular}{|c|c|c|}
\hline Variable & Description & Units/Value \\
\hline time $_{\text {now }}$ & Current time & $h h: m m: s s d d / M M / y y y y$ \\
\hline time $_{\text {leave }}$ & The time the inhabitant left home & $h h: m m: s s d d / M M / y y y y$ \\
\hline time $_{\text {leavePeriod }}$ & The period of time spent away from home & $\min$ \\
\hline time $_{\text {back }}$ & The time of the inhabitant's return home & hh: $: m m:$ ssdd/MM/yyyy \\
\hline time $_{\text {backPeriod }}$ & Time the user spends at home & $\min$ \\
\hline time $_{\text {period }}$ & If today is a working day & [Working $\mid$ NonWorking] \\
\hline time periodDay & Time of day & {$[$ Morning $\mid$ Afternoon $\mid$ Evening $\mid$ Night $]$} \\
\hline periods $_{\text {out }}$ & Number of periods the user spends out of home & {$[1,2, \ldots, n]$} \\
\hline
\end{tabular}

working period, if the inhabitants will be away for more than 10 days or if the heating is turned off completely (this information is obtained by the agents that implement the CBR).

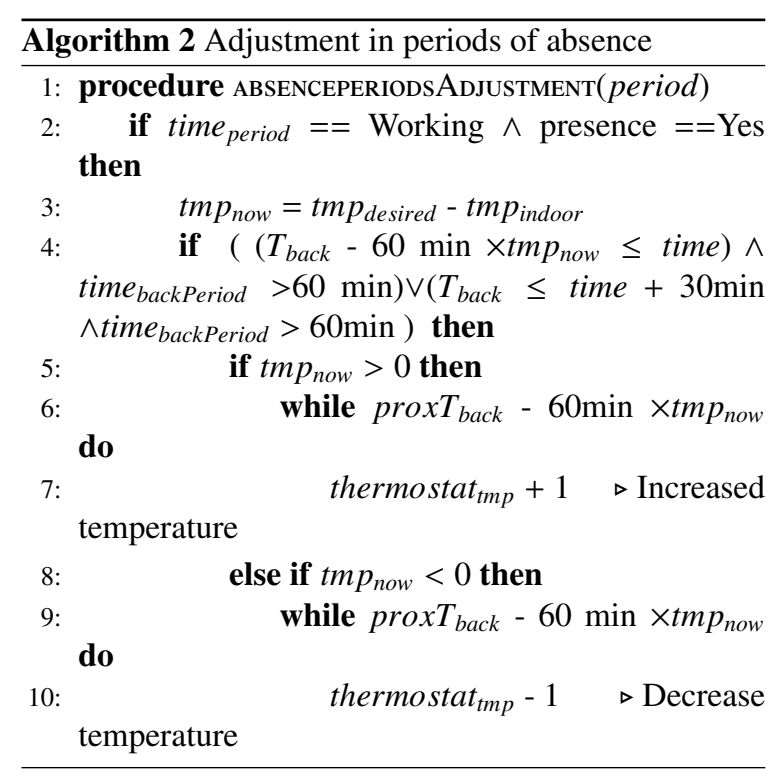

In addition, the previous algorithm is executed in coordination with Algorithm 2, which allows to switch on the heating or increase the temperature when users are about to return home shortly. This algorithm increases or reduces the temperature depending on whether the user is inside or outside the home. Depending on when the user is going to return, the temperature of the home is set accordingly so that by the time the inhabitants arrive the correct temperature is established with the minimum possible consumption. All this is done in the longest time period possible to prevent drastic increases or decreases in temperature over a short period of time, given that this provokes an increase in energy consumption.

\subsection{Gaussian Process Regression for Household En- ergy Consumption}

The architecture has agents (Energy Consumption Prediction Agent in AI Analysis VO) that validate the effectiveness of the algorithms' temperature adjustment decisions. These agents ensure that after the algorithms' decisions are applied, the consumption of energy is reduced. To validate the decisions the agents implement an algorithm that predicts energy consumption using the data collected by the rest of the agents in the architecture. The architecture applies a machine learning model to meteorological data, weather data and the home energy consumption history data to predict future energy consumption. The algorithm performs a training with these data by communicating with agents from other virtual organizations, in this way measuring hourly and daily consumption of electricity.

The chosen machine learning model is Gaussian Process Regression, it predicts energy consumption accurately and in this way allows to validate the effectiveness of the algorithms' temperature adjustments. Lately, Gaussian Process Regression has been frequently used by the machine learning community due to its high accuracy in comparison to other methods, such as Random Forests or Linear Regression.

The agent that implements Gaussian Process Regression uses data collected over an entire year to perform the training process. To avoid a cold start, a crawler agent obtains daily consumption data from the energy supplier. Once data have been collected by the architecture's sensor network for at least six months, it will no longer be necessary for the crawler to obtain daily consumption data. The agent that implements Gaussian Process Regression uses Jython (Python Interpreter in Java) to execute the script that implements the Gaussian Process Regression Algorithm in python using numpy and matplotlib libraries. These libraries are a very useful statistical modeling tool for automated tasks, espe- 
cially since Gaussian Process Regression results are in the form of probability distributions, introducing uncertainty.

\subsection{VAO Architecture}

VOs of agents are a type of distributed architecture in which the agents communicate, coordinate and cooperate to achieve multiple aims such as obtaining sensor data, data analysis through artificial intelligence techniques or the execution of the actions proposed by algorithms. The data obtained by each organization can be sent to any other agent from any other organization that requests them for the organization's internal processes. This makes virtual organizations of agents an ideal approach for the processing of context data coming form various data sources.

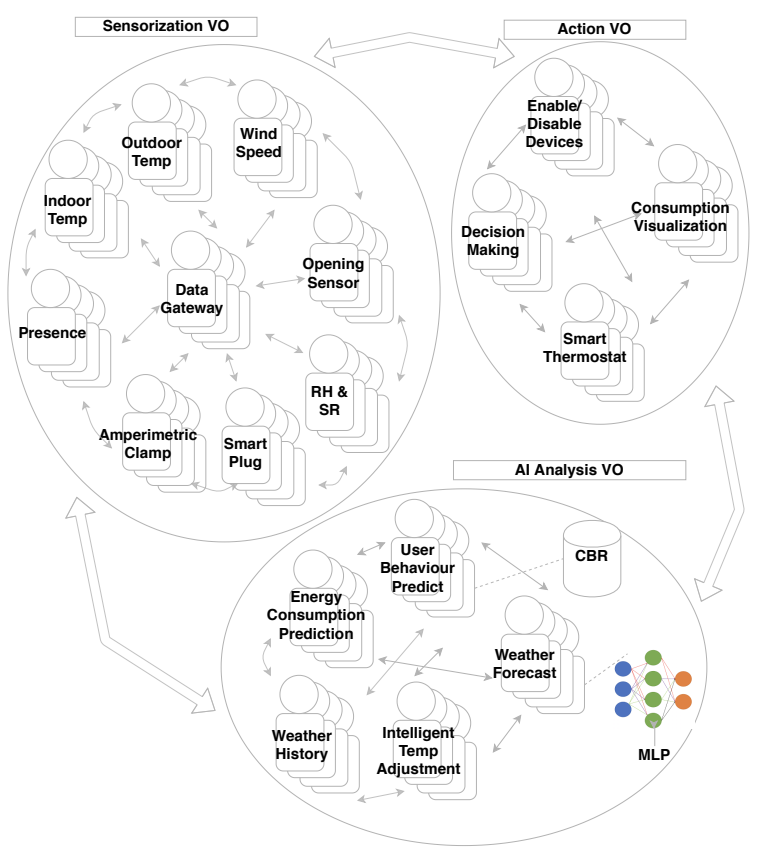

Figure 3: Proposed architecture based on virtual agent organizations for intelligent temperature adjustment by extracting user's behavior characteristics.

The characteristics of virtual organizations of agents make it possible to adopt the artificial intelligence techniques described in the previous subsections. The integration of those techniques will result in a system that feeds on the data processing of each agent. Thus, it is intended to adjust temperature at home in an intelligent way, through the application of algorithms and artificial intelligence techniques in the analysis of the environ- mental data obtained by sensors. The system has four VO, as observed in Fig. 3 .

- Sensor Network VO. This organization's agents are in charge of obtaining the values of the variables that the MLP uses to make a meteorological forecast. These agents also obtain the values of the variables of the CBR cases for the identification of user behaviour models in the dwelling. The values are collected every minute and sent via Z-Wave protocol to the Data Gateway Agent. Data Gateway Agent preprocesses the data in such a way that erroneous values are eliminated. Each agent in this organisation communicates with each of the sensors described in sub-section 3.1

- AI Analysis VO. This organization is the core of system intelligence since it is the organization responsible for all data analysis processes. The system's decisions are made on the basis of those processes. Obtaining the indoor and outdoor temperature values allows to calculate the difference between the temperature at home and the outside temperature. In addition to those values, the solar radiation value is obtained to calculate the temperature that the thermostat should be working at to achieve energy savings. When determining the ideal temperature in the home, it is necessary to consider if people are present at home, since otherwise this temperature may be lower (outside temperature is lower than the inside temperature) or higher (outside temperature is higher than the inside temperature) in order to save even more energy. The decisions of the algorithms are validated by the system thanks to Energy_Consumption_Prediction_Agent

- Action VO: The decisions taken by the AI Analysis VO are sent to the Action VO for intelligent temperature adjustment, once received they are validated by the Decision_Making_Agent using the Gaussian Process Regression. Once the decision has been validated, the temperature is set by means of the Smart thermostat and consumption is monitored through the Smart Plug. This VO contains the Consumption_Visualization_Agent that shows an energy consumption level achieved with the system and the energy that would have been consumed if the system had not been used. Moreover, this VO is responsible for exporting this data in a CSV for subsequent analysis with statistical tools such as SPSS. 


\section{Case Study}

A case study was designed in order to prove the feasibility of the proposed architecture based on virtual organisations of agents, to this end it was implemented in a home [53], [54].

\subsection{Experimental set-up}

The case study was divided into two phases (Baseline period and Evaluation period), each lasted one month: an energy consumption control phase during which the system only monitored variables but did not make any decisions and the evaluation period in which the system could make decisions. The environment in which the functionality of the developed architecture has been evaluated has been carried out in two, northfacing dwellings. A traditional construction house, a flat of $69.98 \mathrm{~m}^{2}$, (Home 1) and a modular house, a flat of $124 \mathrm{~m}^{2}$, (Home 2) constructed by BHS, (http: //www. barcelonahousingsystems.com/es/).

The variables associated with climatological factors are key to the system since they allow it to effectively reduce consumption. In the first part of the optimization algorithm execution, the system uses the values of these variables to adjust the temperature of the HVAC system. In the second part of the execution of the algorithm, the second adjustment is carried out considering the threshold that is established according to the preferences of the home inhabitants. For the two periods (Baseline period and Evaluation period) the values of these variables will be compiled to analyse how the system's actions contribute to the reduction in energy consumption.

The MLP has been trained with meteorological data from October to December 2015 and 2016 (the case study was being performed during those months), as well as the data from the previous 30 days. Since this system had never been used previously, its sensor network had not collected any weather data so these data were acquired by the Weather_history_agent of the AI Analysis VO.

Each of the two periods in which the experiment was divided had a duration of one month. The Baseline period lasted from October 23th, 2017 to November 23th, 2017 and the Evaluation period from November 23th, 2017 to December 23th, 2017. These two periods were chosen to perform the experiment because they have a similar holiday period and their maximum and minimum temperatures barely vary from week to week. Thus, the conditions of the experiment were very similar in the two phases.

The values of the variables in past CBR cases were acquired in the Baseline Period. At this stage, the sys-

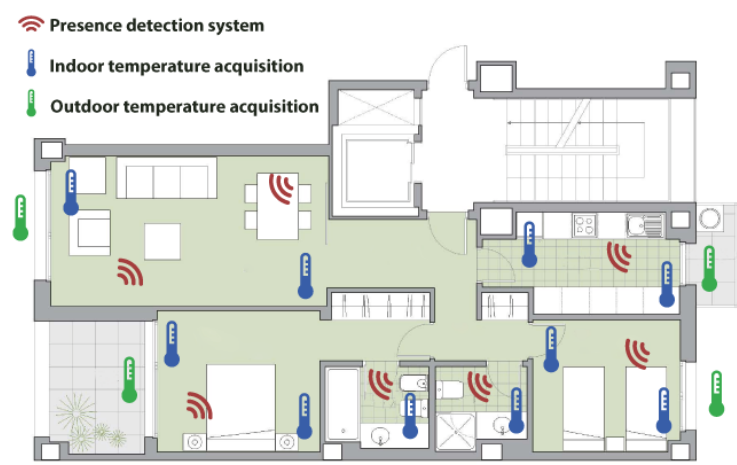

Figure 4: Example of how the sensors were deployed in the traditional construction house (Home 1)

tem stored new cases every day which have been solved successfully for every inhabitant (the annotation of each case was done manually).

Figures 4 and 5 illustrate the distribution of sensors in the case study homes. An outdoor temperature sensor was placed at each window of the house, two sensors in each room, except the two bathrooms where only one was placed, a presence sensor in each room except the living room where two sensors were placed. Averages have been obtained of all the values collected by all the sensors.)

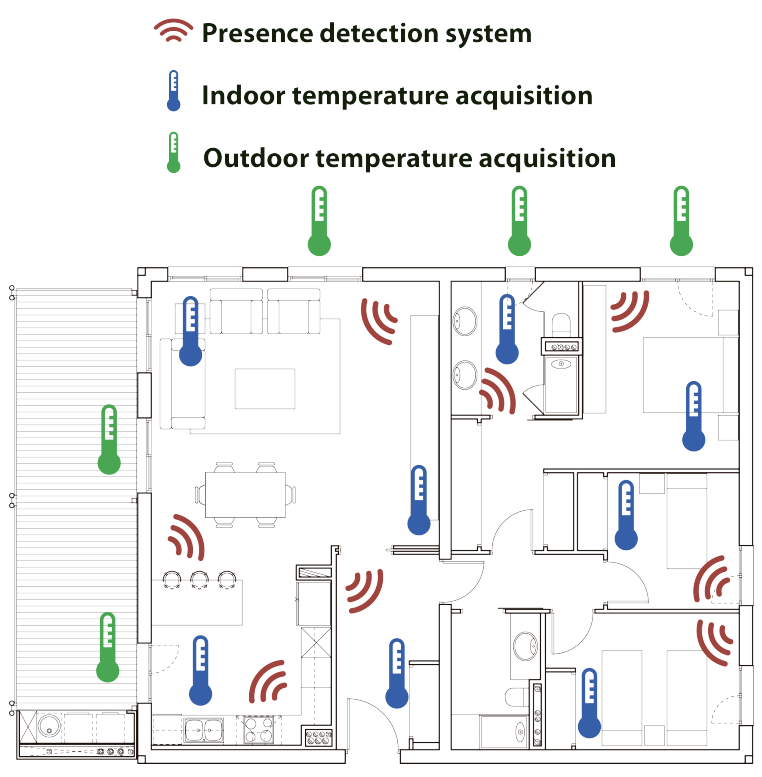

Figure 5: Example of how the sensors were deployed in the modular home (Home 2) 


\subsection{Results}

The following data were collected by the system every 15 minutes: indoor $\left(\right.$ tmp $\left._{\text {indoor }}\right)$ and outdoor $\left(t m p_{\text {outdoor }}\right)$ temperature, presence of people (presence) in the home. This information is managed by the Sensor Network VO thanks to the communication between sensors. The variables used by the optimization algorithm (room type $_{\text {, thermostat }}$ status, thermostat tmp $_{\text {, }}$, time now, time $_{\text {periodDay }}, t m p_{\text {now }}, t m p_{\text {desired }}$ ) are obtained by the AI Analysis VO. The information it uses includes the weather forecast and time period (forecast, time period) and the other variables used by the algorithm, obtained by the Multi-Layer Perceptron (MLP), such as time $_{\text {back }}$, time $_{\text {backPeriod }}$, time $_{\text {leave }}$, time $_{\text {leavePeriod }}$. The rest of the information required by the algorithm is obtained by the Information Management VO. Once the Intelligent Temperature Adjustment Agent (AI Analysis VO) generates a temperature, it must be validated that it will reduce energy consumption.

Table 3: Header of the training dataset.

\begin{tabular}{|c|c|c|c|c|c|}
\hline & weekday & day & week & presence & Whenergy company's website which gives the client's en- \\
\hline 2016-12-01 & 3 & 336 & 48 & 0.3 & $3129.9 \operatorname{tg} y$ use history $(23 / 10 / 2017-23 / 12 / 2017)$. The agent \\
\hline $2016-12-02$ & 4 & 337 & 48 & 0.3 & 2366 . predicts energy consumption at a $95 \%$ confidence inter- \\
\hline $2016-12-03$ & 5 & 338 & 48 & 0.4 & 2388. fal $^{\text {al }}$ and the majority of our predictions are within this \\
\hline $2016-12-04$ & 6 & 339 & 48 & 0.0 & 6029. interval. \\
\hline 2016-12-05 & 0 & 340 & 49 & 0.1 & 2345.0 \\
\hline
\end{tabular}

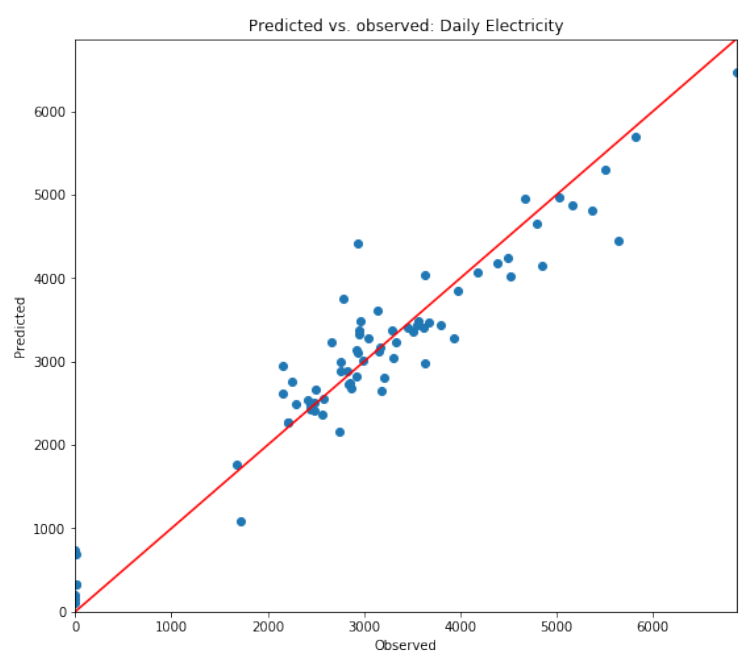

Figure 6: Example of validation of actions (Decision Making Agent); training Gaussian Process Regression (Predicted vs. Observed).

The intelligent temperature adjustment validation process is mainly performed by the Energy Consump-
Table 4: Data collected by the system in each phase.

tion Prediction Agent (AI Analysis VO) and Decision Making Agent (Action VO). The first agent implements the Gaussian Process Regression algorithm, an algorithm that predicts consumption, for which it first performs a training process. In the figure 6 there is an training and testing sample, in which a Training R2 Score has been obtained: 0.8709 and the Test score R2: 0.8163 . Table 3 shows the Header of the training dataset.

Once the classifier has been trained, it is ready to be used to predict future energy consumption. Figure 7 shows the prediction process performed by the Energy Consumption Prediction Agent using Gaussian Process Regression. The results obtained of this agent validated by the Decision Making Agent in the Action VO (The temperature set by the system is considered valid if the consumption loss is lower than the one predicted for that day) prior to running the algorithm. In the validation phase, given that a large dataset was not available, the Energy Consumption Prediction Agent collected a set of consumption data through a crawler script from the

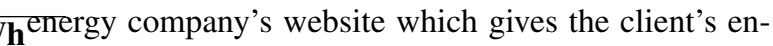
3129.6 gy use history $(23 / 10 / 2017$ - 23/12/2017). The agent 3388. $\mathrm{gal}$ and the majority of our predictions are within this 2345.0

\begin{tabular}{clll}
\hline Period & \multicolumn{1}{c}{ Variable } & Home 1 & Home 2 \\
\hline \multirow{2}{*}{ Baseline } & Avg. Out. Temp $\left({ }^{\circ} \mathrm{C}\right)$ & 9.36 & 11.23 \\
period & Avg. In. Temp $\left({ }^{\circ} \mathrm{C}\right)$ & 24.2 & 23.3 \\
& Energy $(\mathrm{Wh})$ & 128930 & 136370 \\
Evaluation & Avg. Out. Temp $\left({ }^{\circ} \mathrm{C}\right)$ & 6.38 & 7.86 \\
period & Avg. In. Temp $\left({ }^{\circ} \mathrm{C}\right)$ & 20.7 & 21.1 \\
& Energy $(\mathrm{Wh})$ & 90.04 & 118.06 \\
\hline
\end{tabular}

Once the ideal temperature has been established, it is sent to the smart thermostat so that it can be executed. The adoption of these temperatures has allowed to reduce the consumption of energy. In the baseline period, which lasted one month, the system had only collected household data without taking any action. In the evaluation period, the system not only collected the values of all the variables but also made appropriate decisions on the basis of these values, as shown in Table 4 .

Table 5] shows electrical consumption for each stage (Baseline and evaluation period). Table 6 shows the results of the Student's t-test and the Levene test for equality of variances. The difference between the baseline period and the evaluation period is significantly lower with a p-value close to 0.000 . These results demonstrate that 


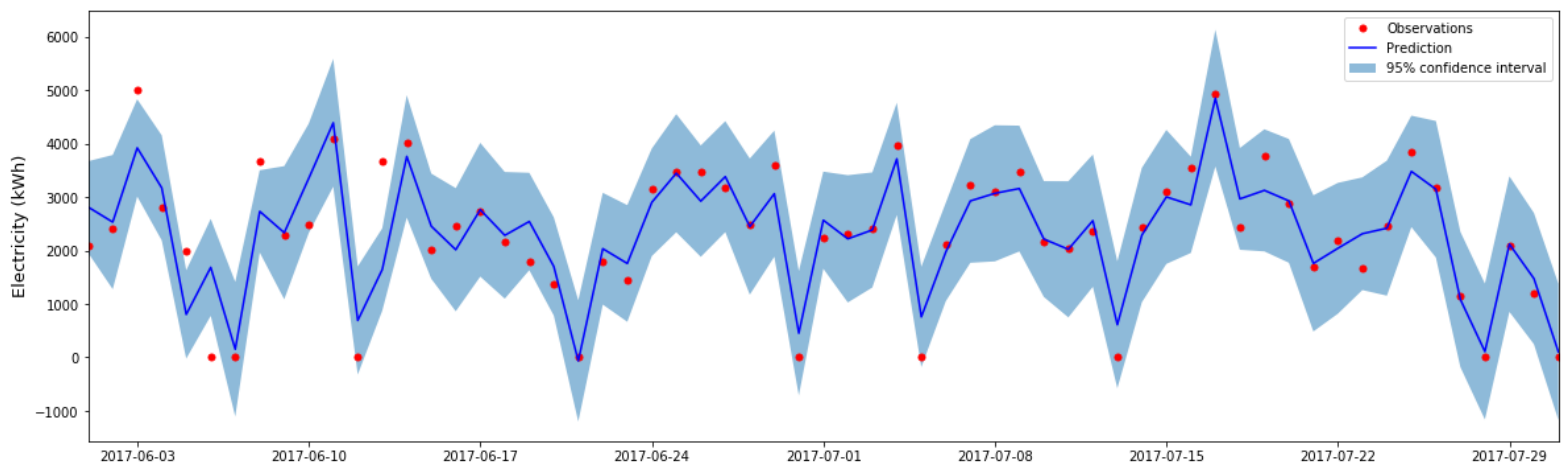

Figure 7: Prediction of future energy consumption using GPR, based on data collected by sensors.

Table 5: Total consumption in Wh in the baseline and evaluation periods, as well as the savings achieved in the second period.

\begin{tabular}{lcccc}
\hline & \multicolumn{2}{c}{ Home 1 } & \multicolumn{2}{c}{ Home 2 } \\
\hline & Without & With & Without & With \\
& system & system & system & system \\
\hline Baseline period (Wh) & 128930 & 128930 & 136370 & 136370 \\
Evaluation period (Wh) & 127000 & 90040 & 141440 & 118060 \\
Difference (Wh) & 1930 & 38890 & -5070 & 18310 \\
Savings (\%) & -1.50 & -30.16 & +3.72 & -13.43 \\
\hline
\end{tabular}

the algorithm achieves energy savings efficiently.

Although the system could achieve a greater reduction in consumption, this would nevertheless affect the added value offered by the system (achieving a reduction in energy consumption by trying not to affect the feeling of comfort by users too much).

There are similar proposals, which like the one presented in this work, manage to reduce energy consumption without affecting the comfort of users. A similar proposal has proven to save at least $10 \%$ energy in deployed systems without affecting customer comfort just by cutting peaks [55]. Our system shows greater savings but does not offer all the functionality such as load shifting e. g., to support combined heat and power generation that this system offers. This is now a commercial product provided by Noda Intelligent Systems AB (www.noda.se), which was formed in 2005 as a spin-off company by the researchers.

The obtained results show how the system is capable of achieving a higher energy optimization percentage ( $30.16 \%$ reduction in energy consumption) in the traditional home compared to the modular home. The cost of the proposed sensors and IoT devices is around 400, reducing the average energy bill of 60 euros by about $25 \%$ (the percentage achieved in both houses) would amortize the cost in two years and three months.

\section{Conclusions}

The presented research work has focused on the development of a system for optimized use of energy in homes and buildings. The developed system is based on virtual organizations of agents and implements intelligent temperature adjustment algorithms which achieve considerable energy savings. The proposal also includes social computing algorithms which make it possible for users to adapt the temperature values according to their preferences. The use of VOs has been the key to achieving the proposed objectives; it is a simple method of deploying the temperature adjustment and social computation algorithms within a single system, it also enables the deployment of agents with different roles: energy consumption prediction, consumption monitoring, extraction of information from sensors or execution of actions (radiators switched on/off or temperature regulation through a smart thermostat).

A case study has been performed in two dwellings (a traditional construction house and a modular home), demonstrating the effectiveness of the presented architecture. The architecture adjusts temperature through 
Table 6: Result of the Student's t-test and Levene's test, performed to assess the difference of means and variances between the Baseline data and the Evaluation period.

\begin{tabular}{cccccccccc}
\hline & Baseline period & \multicolumn{3}{c}{ Evaluation period } & & \\
\hline & Mean & Std. & Mean & Std. & t & p-Value (2-Tailed) & F & p-Value \\
Home 1 & 4297.6667 & 1904.22703 & 2904.5161 & 1096.60640 & 3.516 & 0.001 & 7.933 & 0.004 \\
Home 2 & 4545.6667 & 2207.57893 & 3808.3871 & 1290.40845 & 1.599 & 0.115 & 10.474 & 0.002 \\
\hline
\end{tabular}

an smart thermostat, this prevents drastic changes in the temperature of the house, significantly decreasing electricity consumption. The case study, carried out over a period of two months, shows that the proposed solution achieves a significant reduction in energy consumption. The decision to introduce a modular house in the case study is motivated by the energy efficiency of those houses as opposed to traditional construction houses. The results have demonstrated that the system is able to achieve higher energy optimization $(30.16 \%$ reduction in energy consumption) in a traditional house versus a modular house (13.43\% reduction in energy consumption). As for the results of the algorithms used by virtual agent organizations, the Gaussian Process Regressionbased energy consumption prediction algorithm has had an average accuracy of $87.09 \%$ and the algorithm for calculating user presence in the house has an accuracy of $76.4 \%$.

\section{Acknowledgements}

This work is supported by the Salamanca Ciudad de Cultura y Saberes Foundation under the Atracción del Talento programme (CHROMOSOME project).

\section{References}

[1] M. da Graça Carvalho, Eu energy and climate change strategy, Energy 40 (1) (2012) 19-22.

[2] A. Chiodi, M. Gargiulo, F. Rogan, J. Deane, D. Lavigne, U. K. Rout, B. P. Ó. Gallachóir, Modelling the impacts of challenging 2050 european climate mitigation targets on ireland's energy system, Energy Policy 53 (2013) 169-189.

[3] R. R. Collado, M. T. S. Díaz, Analysis of energy end-use efficiency policy in spain, Energy Policy 101 (2017) 436-446.

[4] H. Wilhite, R. Ling, Measured energy savings from a more informative energy bill, Energy and buildings 22 (2) (1995) 145155.

[5] A. González-Briones, J. Prieto, F. De La Prieta, E. HerreraViedma, J. M. Corchado, Energy optimization using a casebased reasoning strategy, Sensors 18 (3) (2018) 865.

[6] A. Al-Daraiseh, E. El-Qawasmeh, N. Shah, Multi-agent system for energy consumption optimisation in higher education institutions, Journal of Computer and System Sciences 81 (6) (2015) 958-965.
[7] S. D. McArthur, E. M. Davidson, V. M. Catterson, A. L. Dimeas, N. D. Hatziargyriou, F. Ponci, T. Funabashi, Multi-agent systems for power engineering applications-part i: Concepts, approaches, and technical challenges, IEEE Transactions on Power systems 22 (4) (2007) 1743-1752.

[8] D. E. O'Leary, D. Kuokka, R. Plant, Artificial intelligence and virtual organizations, Communications of the ACM 40 (1) (1997) 52-59.

[9] A. González-Briones, J. Prieto, J. M. Corchado, Y. Demazeau, Enervmas: Virtual agent organizations to optimize energy consumption using intelligent temperature calibration, in: International Conference on Hybrid Artificial Intelligence Systems, Springer, 2018, pp. 387-398.

[10] A. González-Briones, F. De La Prieta, M. Mohamad, S. Omatu, J. Corchado, Multi-agent systems applications in energy optimization problems: A state-of-the-art review, Energies 11 (8) (2018) 1928.

[11] P. Hoes, J. Hensen, M. Loomans, B. De Vries, D. Bourgeois, User behavior in whole building simulation, Energy and buildings 41 (3) (2009) 295-302.

[12] C. Harris, V. Cahill, Exploiting user behaviour for context-aware power management, in: Wireless And Mobile Computing, Networking And Communications, 2005.(WiMob'2005), IEEE International Conference on, Vol. 4, IEEE, 2005, pp. 122-130.

[13] A. Afram, F. Janabi-Sharifi, Theory and applications of hvac control systems-a review of model predictive control (mpc), Building and Environment 72 (2014) 343-355.

[14] B. Dong, K. P. Lam, Building energy and comfort management through occupant behaviour pattern detection based on a largescale environmental sensor network, Journal of Building Performance Simulation 4 (4) (2011) 359-369.

[15] K. Hoon, B. Kita, G. Marreiros, K. Haengkon, Y. Hyun, C. Ramos, A study on the key management strategy for wireless sensor networks, ADCAIJ: Advances in Distributed Computing and Artificial Intelligence Journal 3 (3) (2014) 43-53.

[16] A. González-Briones, D. Valdeolmillos, R. Casado-Vara, P. Chamoso, J. A. G. Coria, E. Herrera-Viedma, J. M. Corchado, Garbmas: Simulation of the application of gamification techniques to increase the amount of recycled waste through a multi-agent system, in: International Symposium on Distributed Computing and Artificial Intelligence, Springer, 2018, pp. 332343.

[17] F. Silva, C. Analide, Tracking context-aware well-being through intelligent environments, ADCAIJ: Advances in Distributed Computing and Artificial Intelligence Journal 4 (2) (2015) 6172.

[18] T. L. van Kasteren, G. Englebienne, B. J. Kröse, Human activity recognition from wireless sensor network data: Benchmark and software, in: Activity recognition in pervasive intelligent environments, Springer, 2011, pp. 165-186.

[19] D. Coakley, P. Raftery, M. Keane, A review of methods to match building energy simulation models to measured data, Renewable and sustainable energy reviews 37 (2014) 123-141.

[20] A. Pimenta, D. Carneiro, J. Neves, P. Novais, A neural network to classify fatigue from human-computer interaction, Neuro- 
computing 172 (2016) 413-426.

[21] S. B. A. Kamaruddin, N. A. M. Ghanib, C.-Y. Liong, A. A. Jemain, Firearm classification using neural networks on ring of firing pin impression images, ADCAIJ: Advances in Distributed Computing and Artificial Intelligence Journal 1 (3) (2012) 2734.

[22] A. Rivas, P. Chamoso, A. González-Briones, J. Corchado, Detection of cattle using drones and convolutional neural networks, Sensors 18 (7) (2018) 2048.

[23] B. Dong, B. Andrews, K. P. Lam, M. Höynck, R. Zhang, Y.-S. Chiou, D. Benitez, An information technology enabled sustainability test-bed (itest) for occupancy detection through an environmental sensing network, Energy and Buildings 42 (7) (2010) 1038-1046.

[24] J. Page, D. Robinson, N. Morel, J.-L. Scartezzini, A generalised stochastic model for the simulation of occupant presence, Energy and buildings 40 (2) (2008) 83-98.

[25] V. L. Erickson, M. Á. Carreira-Perpiñán, A. E. Cerpa, Occupancy modeling and prediction for building energy management, ACM Transactions on Sensor Networks (TOSN) 10 (3) (2014) 42.

[26] C. M. Stoppel, F. Leite, Integrating probabilistic methods for describing occupant presence with building energy simulation models, Energy and Buildings 68 (2014) 99-107.

[27] A. Mahdavi, F. Tahmasebi, Predicting people's presence in buildings: An empirically based model performance analysis, Energy and Buildings 86 (2015) 349-355.

[28] A. González-Briones, G. Hernández, T. Pinto, Z. Vale, J. M. Corchado, A review of the main machine learning methods for predicting residential energy consumption., in: 2019 16th International Conference on the European Energy Market (EEM), IEEE, 2019, pp. 1-6.

[29] U. Kumar, V. Jain, Time series models (grey-markov, grey model with rolling mechanism and singular spectrum analysis) to forecast energy consumption in india, Energy 35 (4) (2010) 1709-1716.

[30] L. Ekonomou, Greek long-term energy consumption prediction using artificial neural networks, Energy 35 (2) (2010) 512-517.

[31] A. Azadeh, S. Ghaderi, S. Sohrabkhani, Annual electricity consumption forecasting by neural network in high energy consuming industrial sectors, Energy Conversion and management 49 (8) (2008) 2272-2278.

[32] A. Al-Amoudi, L. Zhang, Application of radial basis function networks for solar-array modelling and maximum power-point prediction, IEE Proceedings-Generation, Transmission and Distribution 147 (5) (2000) 310-316.

[33] J.-D. Wu, J.-C. Liu, A forecasting system for car fuel consumption using a radial basis function neural network, Expert Systems with Applications 39 (2) (2012) 1883-1888.

[34] V. Ş. Ediger, S. Akar, Arima forecasting of primary energy demand by fuel in turkey, Energy Policy 35 (3) (2007) 1701-1708.

[35] J. Contreras, R. Espinola, F. J. Nogales, A. J. Conejo, Arima models to predict next-day electricity prices, IEEE transactions on power systems 18 (3) (2003) 1014-1020.

[36] B. Dong, C. Cao, S. E. Lee, Applying support vector machines to predict building energy consumption in tropical region, Energy and Buildings 37 (5) (2005) 545-553.

[37] R. K. Jain, K. M. Smith, P. J. Culligan, J. E. Taylor, Forecasting energy consumption of multi-family residential buildings using support vector regression: Investigating the impact of temporal and spatial monitoring granularity on performance accuracy, Applied Energy 123 (2014) 168-178.

[38] K. Li, H. Su, J. Chu, Forecasting building energy consumption using neural networks and hybrid neuro-fuzzy system: A comparative study, Energy and Buildings 43 (10) (2011) 2893-2899.
[39] G. Shi, D. Liu, Q. Wei, Energy consumption prediction of office buildings based on echo state networks, Neurocomputing 216 (2016) 478-488.

[40] J. Chen, J. Yang, J. Zhao, F. Xu, Z. Shen, L. Zhang, Energy demand forecasting of the greenhouses using nonlinear models based on model optimized prediction method, Neurocomputing 174 (2016) 1087-1100.

[41] V. Bianco, O. Manca, S. Nardini, Electricity consumption forecasting in italy using linear regression models, Energy 34 (9) (2009) 1413-1421.

[42] L. Pérez-Lombard, J. Ortiz, C. Pout, A review on buildings energy consumption information, Energy and buildings 40 (3) (2008) 394-398.

[43] J. W. Taylor, R. Buizza, Using weather ensemble predictions in electricity demand forecasting, International Journal of Forecasting 19 (1) (2003) 57-70.

[44] B. Yan, X. Li, W. Shi, X. Zhang, A. Malkawi, Forecasting building energy demand under uncertainty using gaussian process regression: Feature selection, baseline prediction, parametric analysis and a web-based tool, in: Proceedings of the 15th IBPSA Conference, San Francisco, CA, USA, 2017, pp. 7-9.

[45] P. Chamoso, A. González-Briones, A. Rivas, F. De La Prieta, J. M. Corchado, Social computing in currency exchange, Knowledge and Information Systems (2019) 1-21.

[46] S. D. McArthur, E. M. Davidson, V. M. Catterson, A. L. Dimeas, N. D. Hatziargyriou, F. Ponci, T. Funabashi, Multi-agent systems for power engineering applications-part 2: technologies, standards and tools for building multi-agent systems, IEEE Transactions on Power Systems 22 (4) (2007) 1753-1759.

[47] Ó. García, R. S. Alonso, J. Prieto, J. M. Corchado, Energy efficiency in public buildings through context-aware social computing, Sensors 17 (4) (2017) 826.

[48] L. Hernández, C. Baladron, J. M. Aguiar, B. Carro, A. SanchezEsguevillas, J. Lloret, D. Chinarro, J. J. Gomez-Sanz, D. Cook, A multi-agent system architecture for smart grid management and forecasting of energy demand in virtual power plants, IEEE Communications Magazine 51 (1) (2013) 106-113.

[49] Z. Wang, R. Yang, L. Wang, Multi-agent control system with intelligent optimization for smart and energy-efficient buildings, in: IECON 2010-36th Annual Conference on IEEE Industrial Electronics Society, IEEE, 2010, pp. 1144-1149.

[50] J. Cai, D. Kim, V. K. Putta, J. E. Braun, J. Hu, Multi-agent control for centralized air conditioning systems serving multizone buildings, in: American Control Conference (ACC), 2015, IEEE, 2015, pp. 986-993.

[51] A. Clausen, A. Umair, Y. Demazeau, B. N. Jørgensen, Agentbased integration of complex and heterogeneous distributed energy resources in virtual power plants, in: International Conference on Practical Applications of Agents and Multi-Agent Systems, Springer, 2017, pp. 43-55.

[52] A. Aamodt, E. Plaza, Case-based reasoning: Foundational issues, methodological variations, and system approaches, AI communications 7 (1) (1994) 39-59.

[53] I. Committee, et al., International performance measurement and verification protocol: Concepts and options for determining energy and water savings, volume i, Tech. rep., National Renewable Energy Lab., Golden, CO (US) (2001).

[54] A. Guerrisi, M. Martino, M. Tartaglia, Energy saving in social housing: An innovative ict service to improve the occupant behaviour, in: Renewable Energy Research and Applications (ICRERA), 2012 International Conference on, IEEE, 2012, pp. $1-6$.

[55] P. Davidsson, Intelligent transport and energy systems using agent technology., in: SCAI, 2013, pp. 3-10. 